\title{
Grippe aviaire et barrières sanitaires : comment les acteurs nationaux se saisissent des opportunités offertes par une crise sanitaire mondiale (cas du Sénégal)
}

\author{
Yacine NGOM*, Guillaume DUTEURTRE**, Abdoulaye NIANG***, \\ Marcel DJAMA** \\ * Institut Sénégalais de Recherches Agricoles (ISRA), SN- Dakar, SENEGAL \\ ** CIRAD, F-34398 Montpellier, France \\ *** Université Gaston Berger (UGB), SN- Saint-Louis, Sénégal \\ e-mail:yacinengom@gmail.com
}

Résumé - Suite à la libéralisation des échanges qui s'est accentuée en 2000, certains pays ont publié des réglementations nationales visant à limiter les importations de produits d'origine animale. Toutefois, il n'a pas été clairement démontré que ces instruments de politique avaient été liés à des objectifs sanitaires, ou à d'autres logiques sociales, politiques ou économiques. Au Sénégal, une mesure d'interdiction des importations des produits de l'aviculture et de matériels avicoles usagers, officiellement justifiée par des garanties sanitaires dans le contexte de la crise de la grippe aviaire, avait été prise en 2005. Cependant, cette mesure a soulevé des débats importants au sein des pays de l'OMC du fait que certains acteurs nationaux et pays exportateurs de viande de volaille la considèrent comme une mesure protectionniste. Ce constat peut amener à mettre l'accent sur les acteurs et la multiplicité des intérêts nationaux et même internationaux en concurrence, dans ces processus. L'objet de cet article est d'expliciter la manière dont les risques et les incertitudes sanitaires peuvent être instrumentalisés par divers acteurs.

L'approche instrumentale de l'action publique (Lascoumes et Le Galès, 2004) a permis d'appréhender cet objet. Cette recherche a été menée entre 2009 et 2010 sur la base d'entretiens conduits auprès des acteurs économiques (aviculteurs, importateurs, provendiers, accouveurs), les services étatiques et la société civile (les associations d'aviculteurs, de commerçants, d'industriels, de provendiers, les interprofessions, les ONG, les associations de consommateurs, etc.) et de données statistiques, documents officiels et communiqués de presse, etc.

L'analyse des résultats montre qu'a priori, la mesure de suspension des importations de produits et matériels avicoles découle de la propagation de la grippe aviaire au niveau de certains pays exportateurs. Cependant, l'analyse du processus dans une perspective diachronique montre que le processus a été initié par les actions menées par les organisations interprofessionnelles, les associations de consommateurs et les industries nationales. L'analyse laisse apparaître l'importance des idées, des règles, et même des stratégies qui sont à la base des actions des acteurs. Elle a également permis de comprendre le positionnement de ces acteurs par rapport aux discours dominants et aux règles commerciales.

Mots-clés : acteurs, politiques publiques, commerce international, viande de volaille, sécurité sanitaire, Sénégal 


\title{
Avian influenza and health barriers: How national stakeholders seize opportunities offered by a global health crisis (case of Senegal)
}

\begin{abstract}
Following trade liberalization which increased in 2000, some countries have developed national regulations to limit imports of products from animals. However, it has not been clearly demonstrated that these policy instruments are linked to health issues, or other social, political or economic logics. In Senegal, a ban on imports of poultry products and poultry equipment users, officially justified by health safeguards in the context of avian influenza crisis, have been implemented in 2005. However, this measure raised concerns among WTO members. Indeed, some national stakeholders and poultry exporting countries saw it as a protectionist measure. This may lead to question the role of stakeholders and the multiplicity of national interests and even international competition in these processes. The purpose of this article is to explain how health risks and uncertainties can be manipulated by various stakeholders.

The instrumental approach of public action (Lascoumes and Le Galès, 2004) helped to apprehend the object. This research was conducted between 2009 and 2010 on the basis of interviews conducted on economic actors (poultry farmers, importers, poultry feed manufacturers, hatcheries), government services and civil society (poultry farmers, traders and poultry feed manufacturers associations, interprofessional, NGOs, consumer associations, etc.) and statistical data, official documents and press releases, etc.

Results show that the ban on imports of poultry products and poultry equipment users has been motivated by the increase of avian influenza in some exporting countries. However, when we analyze the process in a diachronic perspective, we find that it was initiated by the actions taken by interprofessional organizations, consumer associations and domestic industries. The analysis also reveals the importance of ideas, rules and strategies that underlie stakeholders' actions. It further helped to understand the position of these stakeholders in relation to dominant discourses and trade rules.
\end{abstract}

Keywords: Stakeholders, public policies, international trade, poultry meat, food safety, Senegal

Classification JEL : F13, I18

\section{Introduction}

Les dernières décennies ont été marquées par la multiplication de mesures sanitaires affectant le commerce international de produits animaux. Ces mesures ont été induites par plusieurs crises internationales, telles que la crise de la vache folle dans les années 1990, ou plus récemment celles de la grippe aviaire ou de la grippe porcine (World Bank, 2010). Suite à la propagation de la grippe aviaire dans certains pays au cours de l'année 2005, le Sénégal a pris plusieurs mesures, dont la suspension des importations des produits de l'aviculture et de matériels avicoles usagers. Cet embargo sanitaire fut décidé en décembre 2005, et restait encore en vigueur en juin 2015. Cette mesure a été l'objet de controverses et de contestations de la part de certains pays partenaires qui l'ont considérée comme "protectionniste ». Ce débat peut amener à s'intéresser à la complexité des processus de décision politique qui entourent cette mesure et l'implication des acteurs. Afin de comprendre les processus qui ont conduit à cette mesure de cette suspension et la controverse 
qu'elle a suscitée, il apparaît nécessaire d'en analyser le contexte historique, économique, politique, institutionnel et social.

Cette mesure peut être assimilée à un «instrument de l'action publique ", et analysée à partir de l'approche instrumentale de l'action publique, laquelle met l'accent sur les types d'instruments et les motifs qui président à leur choix ou à leur changement. Les études classiques des instruments s'intéressant à l'identification et à la typologie des instruments (Kirschen, 1964 ; Lowi, 1972 ; Hood, 1986 et Kickert, 1998), sur la base de leur degré de coercition et des ressources engagées, ce qui ne permet guère de rendre compte du changement de ses instruments et leurs résultats. Dans cette perspective, Lascoumes et Le Galès (2004) considèrent que les instruments ne sont pas « de simples choix techniques ", mais qu'ils dissimulent aussi des enjeux politiques et induisent des effets spécifiques indépendants des objectifs poursuivis, et qui structurent l'action publique (Lascoumes et Le Galès, 2004). L'approche instrumentale de l'action publique nous a donc semblé appropriée, pour aborder le processus d'élaboration et de remise en cause de la mesure sanitaire dont il est question ici. Pour cela, nous avons choisi de reconstituer ce processus dans une perspective diachronique afin de mettre en évidence son origine, les différentes positions des acteurs qui ont accompagné sa définition, son adoption et ses transformations. L'objectif n'est pas de faire une analyse normative ou une évaluation en termes de réussite ou d'échec mais de rendre plus visible le comportement et l'action des acteurs et de saisir de manière exhaustive les logiques d'acteurs. Il sera ainsi question d'analyser la manière dont les risques et les incertitudes sanitaires peuvent être instrumentalisés par divers acteurs.

Pour aborder cet objet, des outils des méthodes qualitative et quantitative ont été mobilisés. D'une part, des entretiens ont été réalisés auprès des responsables de la société civile, des structures étatiques et certains acteurs économiques entre 2009 et $2010^{1}$. Le choix des acteurs a été fait de façon raisonnée en ne considérant que les acteurs clés qui sont effectivement impliqués dans les processus d'élaboration et de remise en cause de la mesure de suspension des importations de viande de volaille au Sénégal. D'autre part, les comptes rendus de réunions, les procès-verbaux, les textes de lois sur la

\footnotetext{
1 Entre 2009 et 2010, plusieurs entretiens ont été réalisés avec les importateurs, les industriels et les producteurs de viande de volaille et avec les responsables des structures suivantes basées au Sénégal : la Fédération des Acteurs de la Filière Avicole (FAFA), l'Union Nationale des Acteurs de la Filière Avicole (UNAFA), l'Union Nationale des Commerçants et Industriels du Sénégal (UNACOIS), l'Association des Consommateurs du Sénégal (ASCOSEN), l'Union Nationale des Consommateurs du Sénégal (UNCS), l'OXFAM Grande-Bretagne, le GRET, le Collectif des Importateurs et Distributeurs de Viande de Volaille (CIDVV), la Direction du Commerce Intérieur (DCI), la Direction du Commerce Extérieur (DCE), la Direction Générale des Douanes (DGD), la Direction de l'Élevage (DIREL) et le Centre National d'aviculture (CNA), etc.
} 
mesure de suspension ${ }^{2}$ ont permis de reconstituer le processus d'élaboration et de mise en œuvre de la mesure à partir de 2002. L'analyse de contenu des entretiens et l'analyse documentaire ont eu comme objectif d'identifier les acteurs, leurs actions, leurs idées, les règles et leurs stratégies mobilisés autour de l'élaboration et de la remise en cause de la mesure de suspension des importations de viandes de volaille.

L'accent sera mis d'une part sur les actions des acteurs et leur contexte qui permettront de retracer tout le processus de la prise de décision ; et, d'autre part, sur les idées, les règles et les stratégies qui sont à la base des actions des acteurs.

\section{La grippe aviaire et la mesure de suspension des importations de viande de volaille : un lien non causal ?}

La mobilisation des autorités sanitaires internationales (OMS, OIE et FAO) autour de la grippe aviaire ${ }^{3}$ a commencé après l'émergence du virus H5N1 à Hong Kong en 1997 (Keck, 2013) et s'est intensifiée après la crise du Syndrome Respiratoire Aigu Sévère (SRAS) en Asie en 2003 (Figuié et Fournier, 2010) et la diffusion du H5N1 en Asie, en Europe, au Moyen Orient puis en Afrique où les premières victimes humaines furent déclarées entre 2005 et 2006. Face aux risques d'une possible contamination des volailles sénégalaises et à ses conséquences sur la filière avicole, le Sénégal, à l'instar des autres pays déclarés non infectés, décida de prendre des mesures visant à prévenir cette maladie ${ }^{4}$. En effet, lors du conseil des ministres tenu le

\footnotetext{
${ }^{2}$ Arrêté Interministériel n 7717 du 24 novembre 2005 portant interdiction d'importer des produits de l'aviculture et de matériels avicoles usagers Note de service no 210 DGD/DEL du 16 février 2006 portant interdiction d'importation des produits et matériels avicoles Argumentaire du gouvernement du Sénégal relatif à la mesure d'interdiction de l'importation de volailles, à l'attention de la mission permanente du Sénégal à Genève, 2008 Compte rendu de la réunion du groupe de travail sur l'évaluation de la mesure de suspension d'importer de la viande de volailles, jeudi 4 septembre 2008, DCE Compte rendu de la réunion du groupe de travail sur l'évaluation de la mesure de suspension d'importer de la viande de volailles, vendredi 12 septembre 2008, DCE Compte rendu de la réunion du groupe de travail sur l'évaluation de la mesure de suspension d'importer de la viande de volailles, vendredi 19 septembre 2008, DCE Procès-verbal de la VI ${ }^{\mathrm{e}}$ session de la commission mixte Sénégal-Brésil, 8 et 9 mars 2010 , Dakar Compte rendu de la réunion du sous-comité commerce des produits agricoles, jeudi 30 septembre 2010

3 La grippe aviaire ou l'influenza aviaire est une infection provoquée par des virus grippaux de type A, et en particulier les sous-types $\mathrm{H}$ 5, $\mathrm{H} 7$ et $\mathrm{H} 9$. C'est une maladie infectieuse affectant les oiseaux sauvages ou domestiques. Il peut arriver que des souches hautement pathogènes provoquent une maladie respiratoire chez l'homme. Dans ce cas, la contamination se fait par des contacts rapprochés avec les volailles infectées ou avec des objets contaminés.

4 Arrêté n⿳0 005884/PM du 24 octobre 2005 portant création du Comité National de Prévention et de Lutte contre la Grippe Aviaire - CONAGA
} 
20 octobre 2005, le Président de la République avait fait des recommandations d'ordre préventif contre la grippe aviaire ${ }^{5}$.

Quelques jours après (le 25 octobre 2005), une réunion interministérielle portant sur la grippe aviaire et sur les mesures à engager fut organisée. Elle regroupa la Primature, le Ministère de l'élevage, le Ministère du commerce, le Ministère de l'économie et des finances et le Ministère de la santé. Durant cette réunion, les mesures suivantes furent prises : création d'un Comité National de Prévention et de Lutte contre la Grippe Aviaire ou CONAGA, mise en place des Comités (national, régionaux, départementaux et locaux) de Prévention et de Lutte contre la Grippe aviaire, information et sensibilisation des populations, renforcement du contrôle vétérinaire aux frontières, constitution de stocks de sécurité (vaccins aviaires animaux, médicaments antiviraux, désinfectants), mise à niveau des laboratoires de diagnostic et approvisionnement correct en kits de diagnostic, la mise à disposition de quelques matériels de diagnostic, de prévention et de traitement pour les techniciens ${ }^{6}$ et interdiction d'importations des produits de l'aviculture (volailles vivantes, viandes de volailles entières et découpes de volailles, œufs frais et ovoproduits destinés à la consommation et matériels d'exploitation avicole usagés) et de matériels avicoles usagers. Par contre, il fut décidé que les poussins d'un jour destinés à la production continueraient à être importés sur présentation d'un certificat zoo sanitaire et les oufs à couver sur présentation d'un certificat d'origine et de salubrité (cf. Arrêté interministériel $\mathrm{n}^{\circ} 7717$, novembre 2005). Parmi ces multiples réponses, la mesure de suspension des importations de produits et matériels avicoles, du fait de ses enjeux économiques, politiques et sociaux, a suscité de nombreux débats et controverses de la part des acteurs nationaux et internationaux.

5

- Examen immédiat et délivrance par les services vétérinaires d'un certificat phytosanitaire spécial pour tous les stocks de poulets et dérivés ;

- Acheminement de toute volaille ramassée morte, sous emballage plastique, vers les services vétérinaires les plus proches ;

- Mobilisation des chercheurs sénégalais pour aider à mieux comprendre les mutations génétiques du virus et participer à la recherche d'un vaccin ;

- Étude systématique et immédiate des moyens à mettre en œuvre si jamais la maladie venait à survenir au niveau des parcs d'oiseaux migrateurs ;

- Vigilance des aviculteurs et vaccination systématique de la volaille ;

- Mise en place d'un comité spécial de vigilance chargé de surveiller l'évolution de la maladie ;

- Recherche à titre préventif d'antiviraux ;

- Interdiction d'importation de poulets à titre préventif

${ }^{6}$ Techniciens vétérinaires, de la santé humaine, conservateurs des parcs et agents des eaux et forêts 
Elle fut prise en s'appuyant sur l'Accord portant sur les mesures sanitaires et phytosanitaires ou SPS et sur l'Accord général sur les tarifs douaniers et le commerce ou GATT. Dans l'article 5.7 de l'accord SPS, il est précisé :

Dans le cas où les preuves scientifiques pertinentes seront insuffisantes, un Membre pourra provisoirement adopter des mesures sanitaires ou phytosanitaires sur la base des renseignements pertinents disponibles, y compris ceux qui émanent des organisations internationales compétentes ainsi que ceux qui découlent des mesures sanitaires ou phytosanitaires appliquées par d'autres membres. Dans de telles circonstances, les Membres s'efforceront d'obtenir des renseignements additionnels nécessaires pour procéder à une évaluation plus objective du risque et examineront en conséquence la mesure sanitaire ou phytosanitaire dans un délai raisonnable ${ }^{7}$.

Quant à l'accord du GATT, dont l'objectif principal est la liberté des échanges par l'abaissement des droits de douane et la réduction des restrictions quantitatives ou qualitatives aux échanges, il est composé de règles concrètes ciblant les échanges et de règles d'exceptions préconisant la protection. L'article XX.b de cet accord stipule en effet que tout État membre peut prendre toutes les mesures "nécessaires à la protection de la santé et de la vie des personnes et des animaux ou à la préservation des végétaux ${ }^{8}$. Ce qui va en faire une source de controverses dans les pays de l'OMC exportateurs de viande de volaille ${ }^{9}$. Pour certains acteurs, rien ne permet d'affirmer aujourd'hui que la consommation de la viande de volaille cuite ou des œufs cuits peut contribuer à la transmission de la maladie chez l'homme. Il est même précisé sur le site de l'OMS ${ }^{10}$ que

Dans les zones touchées par des flambées, les volailles et produits de volaille peuvent également être consommés sans danger à condition d'être bien cuits et correctement manipulés pendant la préparation ${ }^{11}$.

\footnotetext{
${ }^{7}$ Cf. http://www.ladocumentationfrancaise.fr/dossiers/omc/d-precaution.shtml (Consultation mars 2014)

$8 \quad C f$. http://www.ladocumentationfrancaise.fr/dossiers/omc/d-gattartXX.shtml\#top (Consultation mars 2014)

9 L'OMS sera d'ailleurs forcée d'intervenir en ces termes pour apporter des précisions : «Dans les zones touchées par des flambées, les volailles et produits de volaille peuvent également être consommés sans danger à condition d'être bien cuits et correctement manipulés pendant la préparation » (cf. http://www.who.int/features/qa/29/fr/index.html) (Consultation octobre 2015).

10 Organisation Mondiale de la Santé.

11 http://www.who.int/features/qa/29/fr/index.html (Consultation octobre 2015)
} 
Dans le but de légitimer cette mesure, le gouvernement du Sénégal a mis en avant, à la fois, la faiblesse des capacités techniques de ses services vétérinaires face à une éventuelle attaque de grippe aviaire et l'insuffisance de sa maitrise de la traçabilité des produits et matériels avicoles usagés.

Dans ce contexte, les enjeux de protection de la santé des volailles et des éleveurs sont d'ordre sanitaire, nutritionnel, social, économique et politique. D'un point de vue sanitaire, la propagation de la maladie peut entraîner la mort d'un grand nombre de volailles des suites de la maladie ou l'abattage à des fins prophylactiques, ce qui priverait les populations déjà pauvres d'une importante source de protéines alimentaires (œufs et poulet). Sur le plan économique et social, la propagation de la grippe aviaire peut conduire à des pertes de production considérables et des pertes de sources de revenus pour les éleveurs les plus vulnérables. De même, la peur de consommer des produits avicoles peut entraîner une baisse considérable des ventes et par conséquent des pertes pour les éleveurs et même les provendiers (fournisseurs d'aliments de volaille). Sous l'angle politique, les charges liées aux coûts des mesures de contrôle peuvent s'ajouter aux nombreuses charges financières de l'État sénégalais. Les enjeux de la mesure de suspension des importations de produits de l'aviculture et de matériels avicoles usagers pour les acteurs de la filière avicole peuvent être résumés en deux points essentiels. D'une part, la mesure peut être considérée comme une opportunité pour les aviculteurs, les provendiers (Sédima, Moulins SENTENAC, NMA Sanders, AVISEN, PRODAS, CAM, etc.) et les accouveurs. D'autre part, elle peut être une menace pour les importateurs et les pays exportateurs. Ainsi, les parts de marché de viande de volaille qui étaient détenues par les importateurs seront désormais reprises par les aviculteurs, ce qui peut influer sur les activités des provendiers et accouveurs, qui fournissent des intrants à ces aviculteurs.

Cette situation devait reposer la question sous l'angle des acteurs aussi bien internationaux que nationaux de la filière avicole. Aussi, importe-t-il alors d'appréhender ce processus en mettant en exergue le contexte historique, économique, institutionnel, politique et social de ces acteurs.

\section{Tentative de mise en place d'une mesure de protection de la filière avicole par les organisations professionnelles}

Les débats qui ont opposé dans le passé les acteurs de la filière avicole autour de la mise en place de mesures de restriction aux importations permettent d'éclairer la controverse qu'a suscitée l'embargo décidé dans le cas de la gestion du risque $\mathrm{H} 5 \mathrm{~N} 1$.

En effet, le processus d'élaboration de la mesure de suspension des importations de viande de volaille est étroitement lié à la dynamique organisationnelle des interprofessions de la filière avicole que sont la Fédération des Acteurs de la Filière Avicole (FAFA) et l'Union Nationale des Acteurs de la Filière avicole (UNAFA). 
Le niveau d'organisation et de densification de la filière avicole était étroitement lié à l'évolution administrative, financière et institutionnelle du Centre National d'Aviculture (CNA). Ainsi, la formation et l'encadrement des éleveurs avaient été organisés par le $\mathrm{CNA}^{12}$ depuis sa création en 1962. Après la création du Groupe des Aviculteurs du Sénégal (GAS) en 1964, d'autres associations se sont créées successivement telles que la Coopérative des Aviculteurs du Sénégal (COPAVIS) en 1976, l'Association des Aviculteurs du Cap-Vert (AACV) en 1981 et le Comité Interprofessionnel de l'Aviculture au Sénégal (CIPAS) en 1993.

Ce rôle d'encadrement et de formation de CNA avait été repris par le PRODEC ou Projet de Développement de l'Élevage à Cycle court entre 1995 et 1998, en vue d'appuyer l'organisation et la création d'associations professionnelles ou des regroupements d'associations existantes. Plusieurs associations virent alors le jour entre 1998 et 2001 : l'AAD ${ }^{13}$ (2000), l'AVIDAK $^{14}$ (1999) et le COTAVI ${ }^{15}$ (1998), l'ASCOPA (2000) et l'UNIA $(1999)^{16}$. Cette dynamique, appuyée par le PACEPA ${ }^{17}$ entre 2002-2003, donna naissance à la FAFA dont l'objectif était la promotion de la filière avicole par la mise en place d'un cadre de concertation et de défense des intérêts de la filière. Seule l'association des industriels (UNIA) ne participa pas à la mise en place de cette interprofession à cause de points de désaccords entre producteurs et industriels. Faut-il rappeler que cette période avait coïncidé avec les difficultés de la filière locale, liées à la forte poussée des importations de cuisses de poulet et avec comme conséquence des difficultés de commercialisation des oufs de consommation et de la viande de volaille. La figure (Figure 1) renseigne sur l'évolution des importations de viande de volaille qui sont passées de 272780 à $12405192 \mathrm{~kg}$ entre 1997 à 2003 (ANSD, 2006).

Face aux difficultés de la filière liées à la forte poussée des importations (cf. Figure 1), des concertations avec les acteurs étatiques furent menées et des mouvements de protestation organisés par la FAFA, au courant de cette même année de 2002. Ces protestations aboutirent à la suspension temporaire des importations de viande de volaille et à la suppression de la TVA sur les intrants avicoles au mois d'octobre (Duteurtre et Dieye, 2008). Cette mesure

\footnotetext{
${ }^{12}$ Ce centre a pour mission de contrôler, de coordonner et de superviser toutes les activités avicoles au Sénégal. Il est sous la tutelle de la Direction de l’Élevage (DIREL). Elle a pour activités principales : la formation technique de tous les autres acteurs de la filière et l'enregistrement des données avicoles.

${ }_{13}$ L'Association des Aviculteurs de Dakar regroupe les aviculteurs de la région de Dakar

${ }^{14}$ Créée en 1999, l'Association des Avicultrices de Dakar est composée essentiellement de femmes suite à l'arrêt des activités de la Maison des Aviculteurs.

${ }^{15}$ Le Collectif des Techniciens de Produits Avicoles est fondé en 1998 et regroupe tous les cliniciens, pharmaciens, vétérinaires, techniciens du secteur avicole.

${ }^{16}$ L'Union Nationale des Industriels de l'aviculture.

17 Le projet d'appui à la concertation entre l'État et la profession agricole avait, entre autres, pour mission de regrouper les associations en une interprofession.
} 
Figure 1: Évolution des importations de viande de volaille au Sénégal entre 1996 à 2003 (tonnes)

Source : Agence Nationale de la Statistique et de la Démographie (ANSD), 2006

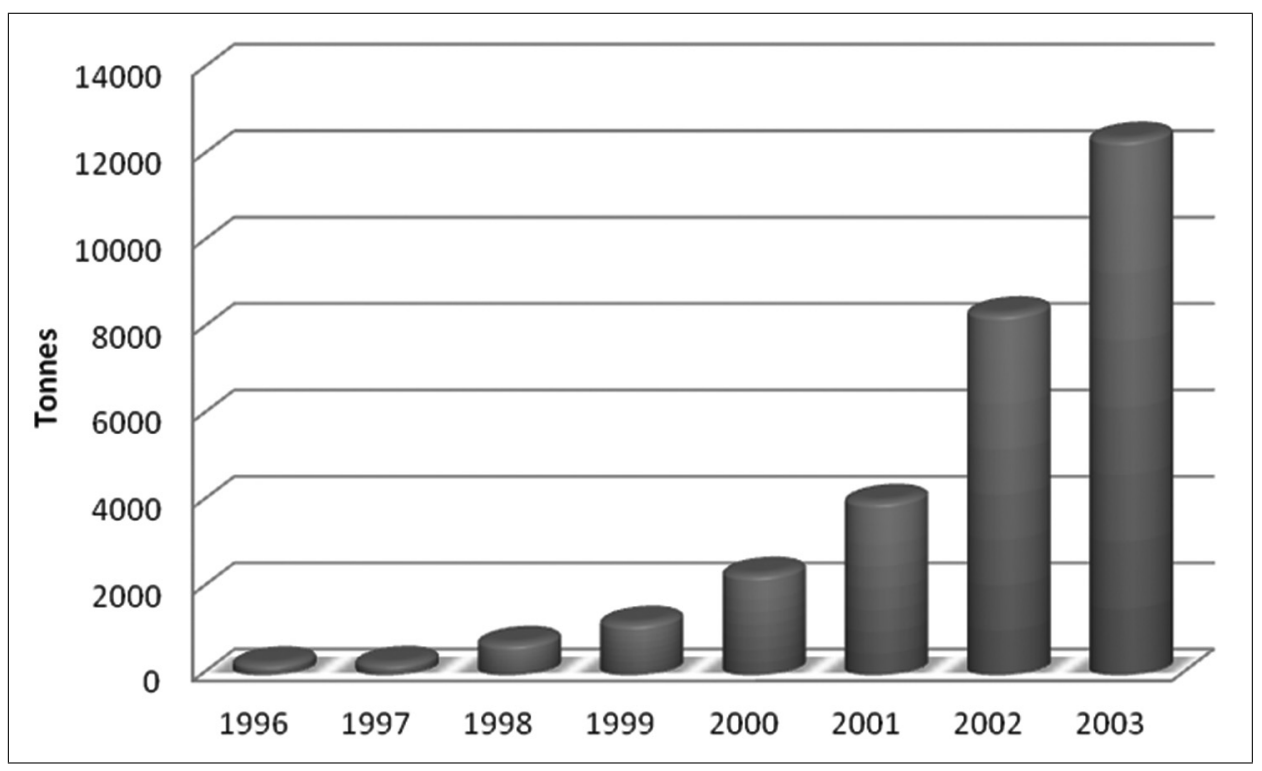

ne fut cependant que temporaire, comme en témoignent les propos d'un des responsables de la FAFA :

La FAFA avait organisé une marche [...]. À la suite de cette marche, la FAFA a obtenu la suspension des importations de viande de volaille au mois d'octobre 2002. $\mathrm{La}$ justification de cette mesure était le principe de précaution. Mais cette justification du Ministère de l'élevage n'était pas assez solide pour permettre de faire perdurer la mesure de suspension. C'est pourquoi elle n'a duré que deux mois (O. L, FAFA, 12 janvier 2010).

Par ailleurs, en raison des désaccords entre industriels et producteurs, les actions de plaidoyers de la FAFA furent menées de manière séparée des initiatives de dialogue entre industries et pouvoirs publics. Ce dialogue industriels-services de l'État visait notamment à sensibiliser les décideurs politiques sur les conséquences néfastes des importations sur l'industrie locale. Ils furent suivis par la rédaction de la part des services du Ministère du commerce de plusieurs notes portant sur la relance de l'industrie avicole au Sénégal (Ministère du Commerce, 2003 et 2005). 
En vue de regrouper les organisations professionnelles et d'harmoniser leurs points de vue, le centre pour le développement de l'entreprise (CDE) ${ }^{18}$, qui était chargé de la mise en œuvre d'un programme du groupe ACP et de la Commission Européenne pour la promotion des investissements et le transfert de technologies dans les entreprises des pays ACP dénommé PROINVEST, engagea un consultant pour mener une large consultation auprès de tous les acteurs de la filière. Ces concertations avaient abouti à la création de l'Union nationale des acteurs de la filière avicole (UNAFA) en 2004. L'UNAFA réunit en son sein une partie des industriels, ainsi que certains membres issus de la FAFA. À partir de ce moment, c'est l'UNAFA qui progressivement porta l'essentiel du plaidoyer anti-importations, parfois aux côtés de la FAFA.

Tandis que des activités de lobbying étaient menées par l'UNAFA auprès des pouvoirs publics en vue de l'instauration de mesures de protection de la filière locale, des actions visant à sensibiliser les consommateurs sur les risques de contamination par la grippe aviaire furent entreprises par l'association des consommateurs du Sénégal (ASCOSEN) ${ }^{19}$.

Dans cette dynamique, lors d'un atelier de partage ${ }^{20}$, l'UNAFA, la FAFA et le COTAVI écrivirent, dans une déclaration conjointe, que le secteur de l'industrie avicole locale était en mesure de fournir aux Sénégalais des poulets de qualité et en quantité suffisante au cas où le Gouvernement déciderait de fermer le pays aux importations avicoles ${ }^{21}$.

Bien sûr, cette décision devait se heurter aux intérêts des importateurs et commerçants de viande de0020volaille dont une partie appartenait à l'UNACOIS (Union Nationale des Commerçants et Industriels du Sénégal). Les commerçants et les importateurs se plaignirent alors du comportement des associations de producteurs et d'industriels qui voulaient se substituer à eux. C'est d'ailleurs ce qui ressort de l'entretien avec l'un des responsables de l'UNACOIS :

Le problème est qu'aujourd'hui, les commerçants ne profitent pas de la production locale car les bénéfices sont trop bas et la production n'est pas très significative. Certains producteurs veulent être en même temps des commerçants (13 janvier 2010, Siège UNACOIS Dakar).

\footnotetext{
18 Son objectif est d'accompagner le développement des entreprises du secteur privé Afrique Caraïbe Pacifique (ACP).

19 Dès 2005, l'ASCOSEN avait commencé à publier toutes les informations relatives à la grippe aviaire sur son site internet en vue de mieux informer les consommateurs sénégalais.

20 Atelier de partage organisé par l'UNAFA en 2005.

${ }^{21} \mathrm{http} / /$ www.ascosen.org $/ \mathrm{milnews}$. php? subaction=showfull\&id=1130895358\&archive

$=\&$ start $\_$from $=\& u c a t=5 \&($ Consultation octobre 2015).
} 
Cette position fut reprise régulièrement par les importateurs qui tentèrent de remettre en cause la mesure d'interdiction des importations quelques années après.

De son côté, l'État mit en place un groupe de travail restreint coordonné par le Ministère de l'élevage en collaboration avec le Ministère du commerce (la DCE et la DCI), le Ministère de l'Économie et des Finances (la DGD), en vue d'une relance de la filière avicole locale. Les travaux de ce groupe aboutirent à l'élaboration du document pour la « Relance de la filière avicole au Sénégal : diagnostic et mesures préconisées » en avril 2004. Sur la base de ces travaux et face aux nombreuses sollicitations des interprofessions et des associations de consommateurs, le Premier ministre donna son accord pour l'instauration d'une surtaxe à l'importation et la révision à la baisse des droits de douane sur les intrants et équipements avicoles, dans sa lettre $n^{\circ} 04710$ PM/SGG/PAS/as du 9 septembre 2005. Cependant ces recommandations ne furent pas suivies de mesures concrètes, puisque finalement un arrêté fut promulgué deux mois après portant interdiction des importations de viande de volaille.

Il faut dire que ces actions en faveur des acteurs de la filière locale furent facilitées par la faible dynamique organisationnelle des importateurs de viandes de volaille. En effet, la plupart des grands importateurs comme Keur Mayoro, Poul Trade, SOPRODAL, Entreprises Dame Ndiaye et le CDA n'étaient pas membres d'une organisation professionnelle. Seuls quelques importateurs étaient membres de l'UNACOIS, qui ne fut consultée qu'en fin de processus. C'est sans doute ce qui explique cette perception d'un des responsables l'UNACOIS qui dit :

La mesure a été prise parce qu'il y avait le lobbying des producteurs locaux qui considèrent que les importations de cuisses de poulets constituent un frein pour le développement de la filière locale (13 janvier 2010, Siège UNACOIS Dakar).

Cette décision, considérée comme une opportunité pour les producteurs, est perçue cependant comme une menace pour les importateurs et les pays exportateurs. Il appartient alors de rendre compte des principaux éléments qui ont alimenté les débats et les discussions sur l'embargo dans le cas de H5N1. Ces opportunités et menaces des uns et des autres mues par des intérêts souvent contradictoires auront sans doute des répercussions sur leurs actions durant la mise en ouvre de cette mesure.

\section{Tentatives de remise en cause de la mesure de suspension}

Dans le cas de $\mathrm{H} 5 \mathrm{~N} 1$, alors que les organisations de producteurs locaux paraissaient se satisfaire de la mesure de suspension, les grands exportateurs 
de viandes de volaille vers le Sénégal (l'UE et le Brésil notamment qui détenaient les principales parts de marchés) entamèrent une série d'initiatives destinées à remettre en cause la mesure de suspension, surtout à partir de 2008.

Les délégations officielles de ces deux pays adressèrent à plusieurs reprises des correspondances dont l'objet était de demander au gouvernement du Sénégal de revoir la mesure de suspension, arguant du fait qu'ils étaient chacun indemne de grippe aviaire. D'après ces correspondances, en tant que membre de l'OMC, le Sénégal se devait de respecter les accords devant permettre aux échanges commerciaux de se poursuivre.

Le Brésil incita même les importateurs à créer une association pour mieux défendre leurs intérêts auprès des autorités étatiques. C'est ce qui fut à l'origine de la création en 2008 du Collectif des importateurs et distributeurs de viandes de volaille qui regroupait des importateurs comme CDA, SOLIDIS, SOPRODAL, Ets Dame NDIAYE, SORECI, Ets Samba FALL, Ets Keur Mayoro, SOFIEX, PATISEN, SAPROLAIT, POUL TRADE et Sénégal Free store. Selon le responsable de Poul Trade, "le Brésil nous avait appuyé pour rédiger une lettre en 2008 au Gouvernement avec des argumentaires justifiant la levée de l'embargo, mais cela n'a pas abouti " (Entretien avec M. O., 1er avril 2010, Dakar). L'argumentaire de ces importateurs s'appuyait d'une part sur les effets négatifs de la mesure sur les entreprises d'importation et de distribution de la viande de volaille, et d'autre part sur l'accessibilité de la viande de volaille en termes de prix, de quantités et de qualité. Ainsi, les chiffres fournis par ces importateurs insistaient à la fois sur la disponibilité de la viande de volaille en quantité suffisante surtout à l'approche des fêtes religieuses (Korité, Noël et Fêtes de fin d'année) où la demande augmente et où les prix sont élevés. Les importateurs ont aussi souligné l'impact négatif de la mesure sur les recettes que l'État tirait des importations.

Cependant, il faut dire qu'il n'y avait pas réellement eu de pertes de recettes, sur les importations, qui pourraient pousser l'État à remettre en cause de la mesure ( $c f$. Figure 2). Ainsi, même si la part des liquidations des importations de viandes de volaille sur les liquidations globales était de 1,7\% en 2004, les liquidations globales n'avaient cessé d'augmenter avant et même trois années après la mesure de suspension en passant de 367 à 490 milliards de Francs CFA entre 2004 et 2008.

Conscient du fait que les argumentaires du gouvernement du Sénégal sur le principe de précaution ne pouvaient plus être valables à long terme, et devant les nombreuses sollicitations des importateurs et des pays exportateurs, le Ministère du Commerce prit l'initiative de réunir en septembre 2008 un groupe de travail incluant la participation du Ministère de l'économie et des Finances et celui de l'élevage. Au nombre de quatre, ces réunions, tenues à la direction du commerce extérieur du Ministère du commerce, évaluèrent la 
Figure 2: Évolution des liquidations globales du Sénégal de 2000 à 2009 en Fcfa Source : Direction générale des douanes (DGD), 2010

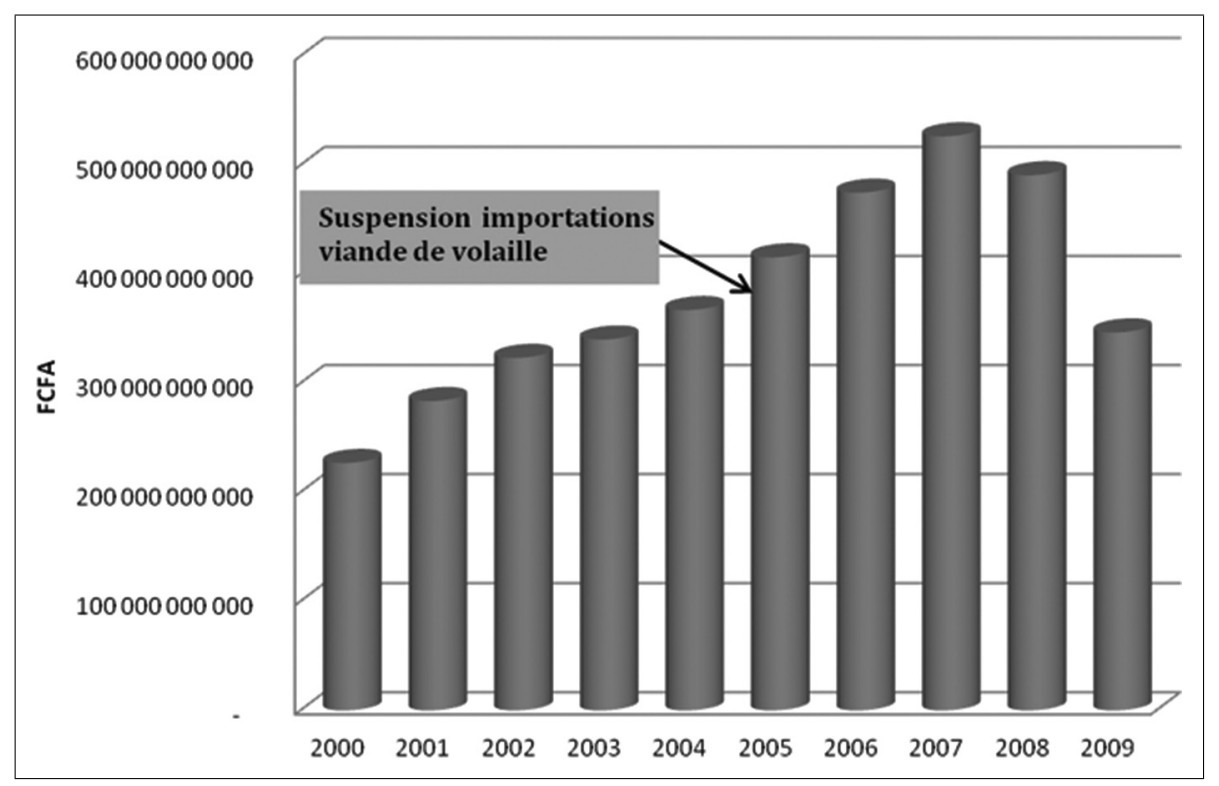

pertinence de la mesure de suspension des importations de viandes de volaille pour raisons sanitaires ${ }^{22}$.

En 2010, le Brésil tenta, de nouveau, de faire connaître ses arguments au gouvernement du Sénégal, mais sans succès. Le compte rendu de la $7^{\mathrm{e}}$ session de la commission mixte Sénégal-Brésil tenue à Dakar les 8 et 9 mars 2010 rend compte de ces discussions :

La partie brésilienne sollicite des Autorités sénégalaises que la mesure interdisant l'importation de volailles et de matériels avicoles soit conforme aux règles de l'Organisation Mondiale de la Santé Animale (OIE) et

22 Cette évaluation préconisait de mettre l'accent sur d'autres arguments pour faire perdurer la mesure ou faire prendre d'autres mesures de protection. D'ailleurs, les quatre réunions tenues ont abouti à l'élaboration d'un document intitulé " politique commerciale en appui à la filière avicole » : ce document, partant du fait que la mesure a influé de façon positive sur la production de la filière et que l'argument sanitaire n'était que provisoire, avait mis l'accent sur la nécessité de fixer d'autres moyens de protection de la filière avicole. Il s'agit de maintenir la mesure au titre de l'accord SPSS (article 5 et 7), de mettre en place une taxation spécifique, d'utiliser les Mécanismes de Sauvegarde (MS) de l'UEMOA et/ou la CEDEAO pour élaborer une taxation spécifique, de trouver un accord sur les quantités maximums à importer, des restrictions sur les abats et des morceaux de poulets pour des raisons sanitaires et de renégocier les droits consolidés conformément à l'article 18 du GATT. En mars 2011, aucune décision n'avait été encore prise pour remplacer la mesure d'embargo sanitaire par de telles mesures de taxation spécifique. 
à l'Accord de Mesures Sanitaires et phytosanitaires de l'Organisation Mondiale du Commerce ( $c f$. Procès-verbal de cette session).

Dans le même texte, pour la partie sénégalaise, le maintien de la mesure se justifie par une faible capacité des services techniques au niveau des contrôles, de la surveillance au niveau des frontières et sur la maîtrise de la traçabilité sur les produits et matériels avicoles usagers.

Suite à cette tentative, le Brésil a décidé de porter l'affaire au niveau du comité chargé des mesures sanitaires et Phytosanitaire (SPS) de l'OMC. En règle générale, même si un pays enfreint une règle de l'OMC, l'organe de règlement des différends n'intervient que si le ou les pays concerné(s) se plaint (plaignent). En d'autres termes, même si l'application de la mesure de suspension des importations n'est pas conforme aux normes de l'OMC, il revient aux pays exportateurs de porter l'affaire au comité SPS.

Face à cette situation, le Sénégal tente d'approfondir les résultats du travail du groupe restreint au sein du Comité National sur les Négociations Commerciales Internationales $(\mathrm{CNNCI})^{23}$, plus précisément au sein du sous-comité commerce des produits agricoles du CNNCI, en vue notamment de tenir compte des préoccupations des acteurs de la filière locale. Le CNNCI sert d'interface entre les acteurs au niveau national et la mission permanente du Sénégal à Genève qui est chargée, entre autres, de suivre les négociations à l'OMC pour le compte du pays. De ce fait, toutes les questions qui concernent la mesure de suspension sont portées à la connaissance de cette mission permanente.

En octobre 2012, les États-Unis (É.-U.) avaient interpellé le gouvernement sénégalais sur la possibilité d'exporter de la viande de volaille au Sénégal ${ }^{24}$. Dans un article intitulé «POULETS-Plaidoyer pour la fin de la restriction des importations : Les Américains veulent approvisionner le marché sénégalais " ${ }^{25}$, paru dans le journal Le Quotidien, l'instance fédérale d'exportation des produits avicoles aux États-Unis (Usapeec) souhaite la levée

23 Créé par le décret n 2001-1072 du 14 décembre 2001, le CNNCI constitue l'outil de formulation et de pilotage stratégique de la politique commerciale extérieure du Gouvernement. Il a été instauré pour définir et défendre les positions du Sénégal dans les négociations commerciales multilatérales, plurilatérales, régionales et bilatérales. Il est composé de plusieurs sous-comités regroupant les représentants des Ministères en charge des questions concernées par les négociations commerciales, le secteur privé et la société civile ( $c f$. Note sur le comité national des négociations commerciales internationales au Sénégal, Direction du Commerce Extérieur, 2003).

24 http://www.setal.net/Les-importations-de-poulets-americains-ne-vont-pas-affecter-lindustrie-avicole-senegalaise_a5990.html (Consultation octobre 2015).

25 http://www.lequotidien.sn/index.php/economie/item/14090-poulets-plaidoyerpour-la-fin-de-la-restriction-des-importations-les-am\%C3\% A9ricains-veulentapprovisionner-le-march\%C3\%A9-s\%C3\%A9n\%C3\%A9galais (Consultation octobre 2012). 
des restrictions à l'exportation de volailles au Sénégal car les États-Unis sont un des rares pays à être indemnes de grippe aviaire.

Durant la période 2012-2013, des concertations et réflexions sur l'élaboration d'un plan de relance durable de la filière avicole ont été entreprises. Lors de la réunion technique interministérielle du 26 juin 2013, les acteurs avaient formulé treize recommandations, dont le maintien de la mesure de suspension de l'importation de produits avicoles pour une période de sept ans (2013-2020), l'application d'une surtaxe au poids sur les viandes de volailles importées, pour une période de trois ans (2021-2023) et la mise en place d'une interprofession avicole. À la suite de cette réunion, l'Interprofession Avicole du Sénégal (IPAS) a été créée lors de l'Assemblée Générale tenue le 2 octobre 2013. L'IPAS regroupe la FAFA, l'UNAFA, la COTAVI, le Directoire des femmes en élevage (DINFEL), l'Union Nationale des Provendiers du Sénégal (UNPS), l'Union Nationale des Accouveurs Professionnels du Sénégal (UNAPS) et l'Association des Grands Producteurs (AGPS). Ainsi, les missions de l'IPAS s'inscrivent dans ce plan de relance de l'aviculture sénégalaise visant à améliorer la compétitivité de l'aviculture sénégalaise en vue de préparer «l'ouverture prochaine des frontières».

En outre, la maladie de la grippe aviaire continue toujours sa progression dans le monde. Selon le bulletin épidémiologique de l'ONMNE du mois de janvier 2011, des cas humains de grippe aviaire avaient été signalés en Égypte, puis un autre au Mexique en juillet $2012^{26}$. En 2015, la grippe aviaire de type H5N1 est apparue au Burkina Faso et en Côte d'Ivoire (avril 2015). Elle est également réapparue en Égypte en janvier 2015. Cette situation laisse des marges de manœuvre au Sénégal qui peut toujours maintenir les mêmes arguments pour la mesure de suspension des importations de viande de volaille.

L'analyse du processus d'élaboration et de remise en cause de la mesure de suspension des importations de viande de volaille révèle une configuration d'acteurs étatiques, de la société civile et économique dont les actions respectives visent à défendre leurs intérêts parfois contradictoires. Ainsi, la mise en place de la mesure de suspension des importations de viande de volaille en 2005 avait été facilitée, la dynamique organisationnelle et la capacité de lobbying des interprofessions (UNAFA et FAFA), des industriels fournisseurs d'intrants avicoles (UNIA) et dans une moindre mesure, des associations de consommateurs (ASCOSEN). Toutefois, une analyse approfondie des actions des acteurs montre que la mesure de suspension des importations de viandes de volaille est d'une part influencée par les idées des ONG, de la recherche et même des organismes internationaux, et, d'autre part, partiellement inféodées aux règles fixées par les accords internationaux et aux stratégies des acteurs. Il

\footnotetext{
26 http://www.lapresse.ca/international/amerique-latine/201207/02/01-4540076-etatdurgence-au-mexique-en-raison-dune-epidemie-de-grippe-aviaire.php (Consultation octobre 2015)
} 
convient alors d'analyser les idées, les règles et des stratégies qui sont à la base des actions des acteurs.

\section{Différentes positions des acteurs dans le processus d'élaboration et de remise en cause de la mesure de suspension des importations de viandes de volaille}

\subsection{Les ONGs et les organisations internationales}

Pour une meilleure vue d'ensemble des idées qui sont à la base des actions des acteurs impliquées dans le processus d'élaboration de remise en cause de la mesure de suspension des importations de viandes de volaille, il nous faut remonter aux pères fondateurs des théories du commerce international en sciences économiques. Ces recherches peuvent être regroupées en trois positions que l'on peut qualifier de dialectique : les défenseurs $\mathrm{du}$ protectionnisme ${ }^{27}$, les partisans du libre-échange ${ }^{28}$ et les auteurs qui développent les arguments en faveur des deux ${ }^{29}$. Le débat sur la protection ou la libéralisation de l'économie a longtemps alimenté les études en économie. Il a aussi été à la base des positions de la plupart des ONG internationales et nationales de développement et des organismes internationaux comme le FMI, la Banque Mondiale et l'OMC. Dans le cadre de cette recherche, les idées et connaissances véhiculées par la recherche en sciences économiques, revient le plus souvent dans l'univers de référence de ces acteurs.

\footnotetext{
${ }^{27}$ Le protectionnisme est une politique économique interventionniste menée par un État. Il vise à protéger de la concurrence internationale, le marché intérieur d'un pays par différentes mesures que sont le droit de douane, les contingentements et autres obstacles non tarifaires variés ( $c f$. Ahmed Silem et Jean-Marie Albertini, 2008, p. 636). Il a été abordé par les mercantilistes (XVIe et XVIIe siècles), les nationalistes (XIXe siècle), les économistes structuralistes, etc.

${ }_{28}^{2}$ La libéralisation est un ensemble de mesures visant à faciliter le développement des échanges commerciaux internationaux, à ouvrir les marchés à la concurrence, en réduisant les tarifs douaniers, les subventions aux producteurs nationaux et à supprimer les contingentements. En sciences économiques, le libre-échange a été développé par des auteurs classiques comme Adam Smith (Recherches sur la nature et les causes de la richesse des nations, 1776), David Ricardo (Des principes de l'economie politique et de l'impôt, 1817), Alfred Marshall (Some aspects of competition, 1890), Paul Samuelson (Protections and Real Wages, 1941), John Stuart Mill (Essays on Some Unsetted and Maximum Revenue Tariffs, 1950), etc. ${ }_{29}$ Cette thèse a été développée par des économistes qui prônent le libre-échange ou le protectionnisme mais qui préconisent la mise en place de mesures protectionnistes ou libérales dans certaines conditions. Autrement dit, aussi bien la libéralisation que la protection a ses limites et il appartient aux pays, par rapport aux opportunités que lui offre telle ou telle branche de l'économie, d'utiliser les deux en vue de meilleurs résultats. Les partisans du libre-échange comme Jean-Baptiste Say (1767-1832), John Maynard Keynes (1883-1946) et ceux du protectionnisme comme Frederick List (1789-1846) ont développé cette thèse.
} 
D'abord, la libéralisation du commerce est plutôt portée par l'OMC, organisme régissant le commerce international. Ensuite, les ONG internationales, en particulier celles qui ont pour mission de contribuer au développement des pays du Sud, ont fortement épousé les thèses protectionnistes. Ces ONG soulignent en particulier l'inéquité des règles du commerce international, eu égard aux subventions accordées aux agriculteurs du Nord et aux mesures protectionnistes de ces pays. C'est le cas des certaines ONG membres de Coordination Sud ${ }^{30}$ comme le GRET, OXFAM France, Agir ICI, le CFSI, ou SYFIA International. Enfin, la troisième position, qui tente de concilier les deux premières positions en vue de meilleurs résultats, avait rarement été mise en avant dans le cadre de processus d'élaboration et de mise en ouvre de la mesure de suspension des importations de viandes de volaille.

De ce fait, les deux premières positions ont aussi alimenté les discussions, sur l'élaboration et à la tentative de remise en cause de la mesure de suspension des importations de viandes de volaille, du côté des acteurs nationaux.

\subsection{Interprofessions}

Durant ce processus, les arguments des interprofessions ont été plutôt économiques, sanitaires et sociaux. Ainsi, selon eux, la protection de la filière avicole avait permis une augmentation des revenus des producteurs, des commerçants, une résorption du taux de chômage des jeunes et une réduction des risques de santé publique liés à l'apparition de la grippe aviaire et au non-respect de la chaîne de froid de l'arrivée du produit importé au port jusqu'aux lieux de consommation.

Influencées par les idées des ONG et de la recherche, les actions des interprofessions comme l'UNAFA et la FAFA ont durant tout le processus mis en avant le discours «protectionniste ». Ils mettaient en avant l'idée d'une protection, la résolution de leur problème de commercialisation, mais aussi la nécessité de fournir au consommateur une bonne qualité de viandes de volaille.

Ces actions visant à protéger la filière locale commencèrent à prendre de l'ampleur à partir de 2004, à la faveur surtout des nombreuses études (Horman, 2004 ; InfoSud Belgique, 2004 ; Sharma et al., 2005 ; Duteurtre et al., 2005 et Diagne, 2004) révélant les impacts négatifs des importations de cuisses de poulets.

\footnotetext{
${ }^{30}$ Elle est la coordination nationale des ONG françaises de solidarité internationale. Créée en 1994, elle rassemble six collectifs et 132 ONG françaises qui mènent des actions humanitaires d'urgence, d'aide au développement, de protection de l'environnement, de défense des droits humains auprès des populations défavorisées et aussi des actions d'éducation de solidarité internationale et de plaidoyer (http://www.coodinationsud.org Répertoire collectifs et ONG membres de Coordination Sud : coordonnées, domaines et pays d'intervention, édition 29 octobre 2010).
} 
Ce sont de telles études qui furent à la base de la campagne, intitulée "Exportations de poulets : l'Europe plume l'Afrique! Campagne pour le droit à la protection des marchés agricoles ", menée par Agir ICI, en collaboration avec le GRET, le CFSI, le CCDF, SOS FAIM et le GRESEA pendant toute l'année 2005. Les organisateurs de cette manifestation précisent alors :

Cette campagne vise à faire reconnaître le droit des pays à protéger leur marché des importations déstructurant leurs filières agricoles locales, afin de mener à bien des politiques en faveur du développement et d'une agriculture durable et solidaire ( $c f$. Exportations de poulets : l'Europe plume l'Afrique! p. 3).

Mais après un premier succès, cette campagne va connaître des difficultés liées à un conflit de leadership entre la FAFA et l'UNAFA, toutes deux pourtant parrainées par Oxfam-GB. La FAFA allait d'ailleurs se retirer de la campagne quelque temps après.

\subsection{Importateurs}

Même si les importateurs avaient adhéré à l'idée de collaborer avec les producteurs de viandes de volaille en vue de la commercialisation de leur produit, ils n'avaient jamais véhiculé l'idée d'une quelconque protection de cette filière. Ainsi, selon le responsable de Keur Mayoro, «la production locale est non seulement insuffisante mais coûte excessivement cher pour le Sénégalais moyen. En réalité le consommateur sénégalais est le plus grand perdant ». Dans ce même ordre d'idées, la lettre adressée aux autorités étatiques par le Collectif des importateurs et distributeurs de viandes de volaille avait insisté sur le fait que les importations facilitaient l'accessibilité en quantités et en termes de prix pour le consommateur sénégalais. Leur stratégie consistait à magnifier les effets positifs d'une libéralisation de la filière comme la satisfaction des besoins des consommateurs.

\subsection{Représentants au Sénégal des pays exportateurs de viande comme le Brésil}

Comme mentionné dans la troisième partie, les représentants du Brésil avaient tenté de remettre en cause la mesure en incitant les importateurs de viandes de volaille à créer une association en vue de défendre leurs intérêts. Dans cette même lancée, les représentants des pays exportateurs (Brésil et les États Unis en particulier) avaient initié des négociations avec les structures étatiques. Ces 
actions étaient fondées sur des stratégies offensives, mais aussi défensives ${ }^{31}$. La décision du Brésil de porter l'affaire au niveau du comité SPS a été guidée par les règles de fonctionnement de l'OMC, dont il est membre.

\subsection{Services étatiques}

Du côté de l'État, même si l'arrêté interministériel portant interdiction d'importations de produits et matériels avicoles était basé sur les règles de l'OMC (principe de précaution et article XX.b du GATT), il est difficile d'unifier la position du gouvernement durant le processus d'élaboration et de remise en cause de la mesure. En effet, les trois Ministères qui ont en charge le dossier ont des rôles spécifiques qui semblent même contradictoires.

D'abord, le Ministère du commerce à travers la direction du commerce intérieure (DCI) a pour mission d'assurer le bon fonctionnement du marché intérieur, de garantir la loyauté, la transparence et la sécurité des consommateurs. De manière plus précise, tout en favorisant et en veillant à la qualité des produits, la DCI doit aussi veiller à l'approvisionnement correct et régulier des villes. En réalité, ces deux objectifs semblent même contradictoires dans la mesure où certaines études et données statistiques (Ngom, 2013 et ANSD, 2006) ont montré que la mesure de suspension des importations de viandes de volaille avait un effet négatif sur les prix au consommateur et par conséquent sur la fréquence de consommation des ménages.

Cependant, durant tout le processus d'élaboration de mise en ouvre de la mesure, le Ministère du Commerce a privilégié l'argument sur la protection de la filière avicole. C'est en tout cas ce que semble indiquer un article paru dans le journal Le Soleil au lendemain de l'atelier organisé par l'UNAFA, en octobre 2005 à la chambre de commerce de Dakar :

Quant au ministre du Commerce, Mamadou Diop «Decroix ». Il a révélé que le Sénégal consacre annuellement près de 12 milliards de francs CFA à l'importation des cuisses de poulets. Il a, en outre, rappelé les décisions prises par les autorités dont la fermeture des frontières maritimes et aériennes et les recommandations adressées au Directeur général de la Douane ${ }^{32}$.

Quant à la Direction du commerce extérieur ou DCE de ce même ministère, qui a pour mission générale de promouvoir le développement des échanges

31 «Les stratégies des acteurs ne sauraient donc se limiter à leurs objectifs clairement définis et à leurs projets cohérents mais comme un jeu, contingent au comportement et au vécu du participant. Ainsi, la stratégie de l'acteur revêt deux aspects : offensif pour saisir les opportunités et contraindre, et défensif pour agir et échapper aux contraintes » (cf. Crozier et Friedberg, 1977, p. 56).

$32 \mathrm{http}: / /$ www.lesoleil.sn/article.php3?id_article=4968 (Consultation mars 2014). 
extérieurs et de mettre en place des mécanismes favorisant l'intégration des entreprises dans le commerce extérieur, elle développa un argumentaire fondé essentiellement sur les règles de fonctionnement de l'OMC. Ainsi, dans le compte rendu de la réunion du groupe de travail du 19 septembre 2008, il est mentionné que «[...], le Conseiller technique a indiqué qu'il paraissait d'autant plus difficile, à ce jour pour le Sénégal, de maintenir une interdiction des importations de produits avicoles pour motifs sanitaires qui frapperaient l'ensemble des pays, y compris ceux qui peuvent prouver qu'ils sont indemnes de grippe aviaire».

Ensuite, le Ministère de l'Économie et des Finances, à travers la Direction Générale des Douanes (DGD), qui est chargée de la perception des droits et taxes douanières, en ayant l'obligation de fournir des informations sur les importations en vue d'une meilleure évaluation de la mesure et de vérifier si la mesure n'aura pas des effets négatifs sur les liquidations globales, avait également une position institutionnelle basée sur les règles de fonctionnement de cette direction.

Enfin, le Ministère de l'élevage, à travers la direction de l'élevage (DIREL) et la direction des services vétérinaires (DSV), est chargé d'apporter son concours pour toutes les questions concernant l'élevage. Il doit non seulement contrôler l'origine et la salubrité des produits et sous-produits animaux, mais aussi proposer des mesures assurant le développement des filières. Ce qui justifie d'ailleurs l'accompagnement des interprofessions (FAFA, UNAFA et IPAS) dans leurs réflexions pour la relance de cette filière ${ }^{33}$ et aussi ses activités de mise en œuvre des mesures de biosécurité : renforcement du contrôle vétérinaire aux frontières, constitution de stocks de sécurité (de vaccins humains et aviaires, de médicaments antiviraux, etc.) et mise en place d'un schéma de surveillance épidémiologique (aussi bien chez les oiseaux que chez l'homme) et d'alerte précoce. À cet effet, le comité national de lutte contre la grippe aviaire (CONAGA), assurera la mise en œuvre de ses mesures de biosécurité. Cependant, l'évaluation du CONAGA en 2008 révélera une faible capacité des services techniques concernant le contrôle sanitaire au niveau des frontières, la surveillance épidémiologique de la maladie et la prise en charge d'une éventuelle crise. C'est pourquoi, l'UE va appuyer alors le gouvernement du Sénégal dans la lutte contre la grippe aviaire, à travers notamment le PAPLUGA (Programme commun d'Appui au Plan national de LUtte contre la Grippe Aviaire ${ }^{34}$ ). En définitive, l'analyse des discours des acteurs étatiques, des acteurs de la société civile et des acteurs économiques,

\footnotetext{
33 Cet accompagnement mettait en avant l'idée de protéger ces interprofessions.

34 Sous-tutelle du Ministère de l'élevage, ce programme vise à limiter les risques de pandémie humaine par le renforcement de la capacité des Services vétérinaires dans la prévention, la détection précoce et le contrôle de la grippe aviaire, ainsi que l'information et la sensibilisation des populations. La coordination est assurée par la direction des services vétérinaires.
} 
montre les différentes positions par rapport à la mesure de suspension des importations décidée en 2005.

\section{Discussions}

Les résultats de ce papier ont montré que les acteurs économiques et la société civile se sont appropriés les connaissances scientifiques véhiculées par la recherche et les ONG. Les positions sur les atouts et les limites du libre-échange sont très manifestes dans la filière viande de volaille au Sénégal. Celle-ci a connu deux options de politique commerciale.

L'option de libéraliser sans soutien aux aviculteurs avait été appliquée entre 2000 et 2005. Cette option avait eu comme conséquence une forte poussée des importations de découpes et de poulets entiers et une faible production de volaille au Sénégal (cf. Duteurtre et al., 2005).

La deuxième avait commencé avec la mesure de suspension des importations de viandes de volaille en 2005 et se poursuit jusqu'à nos jours (juin 2015). Les résultats ont montré que c'est cette dernière option que les acteurs de la filière locale et les services étatiques veulent maintenir (DIREL, DCE). De plus, lors de la rencontre avec le groupe Afrique Caraïbe et Pacifique (ACP) tenue le 17 octobre 2012, le discours du Président de la République du Sénégal Monsieur Macky Sall semble s'inscrire dans cette lancée. En effet, le Président de la République du Sénégal avait réagi à cette question sur la mesure de suspension des importations de viandes de volaille en ces termes :

[...] Doit-on sacrifier encore ces aviculteurs qui contribuent à la richesse nationale et qui par leurs efforts personnels ont pu relancer une filière ? [...] ce sont les vraies questions qui se posent à nos économies et qui sont des questions sur lesquelles nous devons continuer à réfléchir avec nos partenaires tout en préservant bien entendu nos intérêts $[\ldots]^{35}$

La question sur le maintien ou la suppression de la mesure de suspension des importations de viandes de volaille est toujours d'actualité. Aujourd'hui (juin 2015), même si la mesure de suspension des importations de viandes de volaille semble être assez instable sous la pression des pays exportateurs comme le Brésil, l'UE et les États-Unis, la grippe aviaire est apparue récemment au Burkina Faso et en Côte d'Ivoire (avril 2015) et en Égypte (janvier 2015). Cette situation laisse des marges de manœuvre au Sénégal, qui peut toujours maintenir les mêmes arguments (arguments sur le principe de précaution et l'article XX.b du GATT) pour cette mesure. Cependant, ces arguments n'étaient que provisoires. Ainsi, le Sénégal aura-t-il la possibilité de maintenir

35 Journal de 20h de la RTS du mercredi 17 octobre 2012. 
cette mesure avec la disparition de la grippe aviaire dans ces pays ? Aura-t-il la possibilité de ne pas respecter les accords signés dans le cadre de l'OMC ?

En tout état de cause, la forte influence des règles semble montrer les faibles marges de manœuvre du Sénégal par rapport à ces accords. En effet, le Sénégal a toujours été membre de plusieurs entités (UEMOA, OMC, CEDEAO, etc.) qui ont fortement influencé les décisions commerciales.

L'analyse du processus montre aussi une forte influence des stratégies des acteurs. Les acteurs avaient adopté des «stratégies offensives " et des «stratégies défensives». Celles-ci étaient plus visibles du côté des interprofessions (UNAFA et FAFA), des acteurs économiques (industriels fournisseurs d'intrants avicoles, importateurs) et des pays exportateurs (Brésil, UE, etc.). Ce papier fait donc ressortir l'une des dimensions (la stratégie) de l'acteur qui agit pour améliorer sa capacité d'action ou pour préserver ses marges de manœuvre. Il confirme l'idée développée par Jean-François BAYART (1989) sur la place déterminante des stratégies individuelles et collectives, du jeu des acteurs et des relations de pouvoir dans l'analyse de l'action publique en Afrique.

Les résultats de l'étude soulèvent des questions sur l'intérêt général. L'intérêt général est-il l'intérêt des consommateurs ou celui des producteurs? Partant du fait que chaque personne est un consommateur, on peut logiquement conclure que l'intérêt général est l'intérêt du consommateur. Toutefois, le fait de mettre en avant l'intérêt du consommateur dans les options de politique commerciale peut ne pas être une solution durable à cause des risques sanitaires ou de volatilité des prix sur le marché mondial comme ce fut le cas en 2008 avec la flambée des prix du lait en poudre et d'autres denrées alimentaires sur le marché mondial (blé, riz, huile, sucre, etc.).

L'enjeu pour l'État serait de concilier ses préoccupations budgétaires, les intérêts des aviculteurs, des consommateurs et la conformité avec les règles de l'OMC, de l'UEMOA et de la CEDEAO. Cela implique des politiques concertées avec des études au préalable. Mais la question reste ouverte : comment concilier ces différentes préoccupations qui semblent $a$ priori contradictoires?

\section{Conclusion}

L'analyse du processus d'élaboration de la mesure de suspension des importations de volaille révèle qu'elle découle a priori de la propagation de la grippe aviaire au niveau des pays exportateurs. Cependant, l'analyse diachronique de cette mesure révèle que l'embargo s'inscrit dans un contexte beaucoup plus global de discussions autour de l'opportunité ou non de protéger l'industrie avicole locale de la concurrence extérieure.

Les effets diversement ressentis par les acteurs sont à l'origine de la tentative de remise en cause ou même de maintien de la mesure. Ainsi, les exportateurs et les importateurs qui se sentaient lésés ont tenté de remettre 
en cause la mesure tandis que l'État, en collaboration avec les acteurs de la filière locale, essaya de la maintenir ou de mettre en place d'autres mesures de protections plus durables de la filière avicole sénégalaise.

L'analyse laisse apparaître l'importance des idées, des règles, et même des stratégies qui sont à la base de leurs actions. En réalité, les idées véhiculées par la recherche en sciences économiques et sociales, les règles de fonctionnement de l'OMC (qui est chargé de la réglementation du commerce), les règles de fonctionnement des structures étatiques et même les stratégies des acteurs ont largement influé sur les actions des acteurs. De ce point de vue, l'analyse nous a permis de comprendre le positionnement de ces acteurs par rapport aux discours dominants et aux règles commerciales.

En définitive, la mesure de suspension des importations de viande de volaille ne peut être interprétée comme une solution techniquement optimale, mais plutôt comme un compromis institutionnel résultant de la confrontation de différentes logiques, dont la portée dépasse le domaine de la santé, et qui se réfere plutôt à la défense de l'intérêt des acteurs impliqués dans le jeu de la concurrence sur le marché de la viande avicole.

Par ailleurs, ce travail de recherche peut alimenter les débats sur la nécessité de prendre en compte les spécificités nationales dans le choix des politiques commerciales prises au niveau régional ou international ${ }^{36}$. En dépit des politiques commerciales établies au niveau régional et international (UEMOA, CEDEAO et OMC), les acteurs étatiques ou non auront toujours des marges de manœuvre qui leur permettent de contourner les règles établies dans ces cadres régionaux ou même internationaux.

\section{Bibliographie}

ANSD (2006) (Agence Nationale de la Statistique et de la Démographie) Situation Économique et sociale du Sénégal (SES), 279 p.

Ici Agir (2005) (Association française de solidarité internationale, devenue Oxfam en 2003) Exportations de poulets : L'Europe plume l'Afrique! Campagne pour le droit à la protection des marchés agricoles, Campagne d'Agir Ici n ${ }^{\circ} 68,, 4$ p.

Bayart J.-F. (1989) L'État en Afrique : la politique du ventre, Paris, France, Fayard, $439 \mathrm{p}$.

\footnotetext{
${ }^{36}$ Les négociations de l'OMC pour déterminer les produits spéciaux et les mécanismes de sauvegarde spéciale, les négociations commerciales entre UE et les pays ACP dans le cadre des APE pour la détermination des produits sensibles et celles de la CEDEAO pour la mise en place du tarif extérieur avec l'établissement d'une $5^{\mathrm{e}}$ bande vont influer sur les politiques commerciales au niveau national.
} 
Crozier M. et Friedberg E. (1977) L'acteur et le système : les contraintes de l'action collective, Paris, France, Seuil, 500 p.

Diagne B. M. (2004) Étude de l'impact économique des importations de poulets entiers et en morceaux sur le développement de la filière avicole au Sénégal, Rapport Final, Oxfam International, Dakar, , 73 p.

Direction du Commerce Extérieur (2003) Note sur le comité national des négociations commerciales internationales au Sénégal, Ministère de l'élevage et du commerce, Dakar, Sénégal, 14 p.

Duteurtre G., Dieye P. N. et Dia D. (2005) Ouverture des frontières et développement agricole dans les pays de l'UEMOA : L'impact des importations de volailles et de produits laitiers sur la production locale au Sénégal, rapport de recherches commandité par la FAO, Institut Sénégalais de Recherches Agricoles, Dakar, Sénégal, 78 p.

Duteurtre G. et Dieye P. N. (eds) (2008) Les organisations interprofessionnelles agricoles au Sénégal : de nouveaux outils de régulation des marchés, Bureau d'analyses macro-économiques de l'Institut sénégalais de recherches agricoles (Isra-Bame), Dakar, Sénégal, 192 p.

Figuié M. et Fournier T. (2010) Risques sanitaires globaux et politiques nationales : la gestion de la grippe aviaire au Vietnam, Revue d'Études en Agriculture et Environnement, 91(3), 327-343.

Horman D. (2004) Chicken Connection. Le poulet africain étouffé par l'Europe : Agrobusiness, dumping, souveraineté alimentaire..., Bruxelles, Belgique, GRESEA, $136 \mathrm{p}$.

Hood C. (1986) The Tools of Government, London, UK, Chatham House Pub, Mac millan, $178 \mathrm{p}$.

InfoSud Belgique (2004) Enquête. Impact des importations de volailles en Afrique de l'Ouest, enquête réalisée pour le Comité Catbolique contre la Faim et pour le Développement réalisée par SYFIA international, URL : bttp://ccfd-terresolidaire.org/IMG/pdflenquetepdf-2261d.pdf, 42 p.

Keck F. (2013) Santé animale et santé globale : la grippe aviaire en Asie, Revue Tiers Monde, 215(3), 35-52.

Kickert W. (1998) Public management and administrative reform in Werstern Europe, Edgard Edward Publishing, London, UK, 278 p.

Kirschen E. (1964) Economic policy in our time, Chicago, USA, Rand Mc Nally and Amsterdam, The Netherlands, North-Holland, 464 p.

Lascoumes P. et Le Galès P. (2004) Gouverner par les instruments, Paris, France, Presses de sciences Po, 370 p.

Lowi T. (1972) Four systems of policy, politics and choice, Public administration review, 32(4), 298-310. 
Ministère du Commerce (2003) Propositions de mesures de soutien pour un développement intégré du commerce dans le secteur de l'agro-industrie " Maïs-aviculture », ministère du Commerce, Dakar, 4 p. et tableau.

Ministère du Commerce (2005) Note d'évaluation sur la mise en application de la suspension des importations de viande de volaille, Division de la consommation et de la qualité, direction du commerce intérieur, $3 \mathrm{p}$.

Ngom Y. (2013) Logiques d'action et analyse diachronique des instruments de la politique commerciale du Sénégal : Mesures de suspension des importations de viande de volaille et de suppression des droits de douane et de la taxe sur la valeur ajoutée sur le lait en poudre, Thèse de Doctorat en Sociologie, Université Gaston Berger de Saint Louis, Sénégal, 364 p.

Sharma R., Nyange D., Duteurtre G., Morgan N. (2005) The impact of import surges: country-case study results for Senegal and Tanzania, FAO Commodity and Trade Policy Research Working Paper $n^{\circ} 11$, URL : ftp://ftp.fao.org/docrep/fao/007/ae520e/ae520e00.pdf, 27 p.

Silem A. et Albertini J.-M. (eds) (2008) Lexique d'économie, 10e édition, Paris, France, Dalloz, $788 \mathrm{p}$.

World Bank (2010) People, Pathogens, and Our Planet; volume 1: Towards a One Health Approach for Controlling Zoonotic Diseases, World Bank Report No. 50833-GLB, Washington, USA, 74 p. 
